\title{
SYSTEMS ANALYSIS AND ECOLOGY ${ }^{1}$
}

\author{
M. B. DALE ${ }^{2}$ \\ School of Natural Resources, University of Michigan, Ann Arbor, Michigan
}

\begin{abstract}
Systems analysis is defined as the use of scientific method with conscious regard for the complexity of the object of study. It has strong relationships with problem solving, in that the same four phases-lexical, parsing, modelling, and analysis-are identifiable in both. Examination of each of these phases reveals some of the problems involved in the use of systems methods in ecology. A model of a precipitation-evaporation system is presented as an example. Problems in experimenting with models of systems and with control, optimization, and comparison of such models are considered.
\end{abstract}

\section{INTRODUCTION}

Systems analysis has been presented as a desirable framework on which the investigation and comparison of ecosystems can be hung. This approach has been especially emphasized by the productivity subgroups of the International Biological Program (IBP). Claims of the importance of systems analysis are not restricted to ecology, for in other fields the results of employing these methods have been claimed to give additional insight and clarity (see e.g. Halmos and Vaughan 1950, Bush and Mosteller 1955, Glanzer and Glaser 1959, Orcutt 1960, Harary and Lipstein 1962, Keeney, Koenig and Zemach 1967). Examples of explicit use of systems methods in ecology are few (Olson 1963, Patten 1965, Holling 1966, Watt 1968), and it is by no means clear from these examples what systems analysis is, what it does, what restrictions it imposes, nor how the variety of ecology (or more precisely ecological methodology) can be attached to this framework. This paper attempts to clarify some of the questions an ecologist must answer and the problems he must resolve before using systems methods, and to introduce some of these methods in the context of a general systems approach. It does not provide the mathematical, statistical, and other details of the use of the methods, although it is hoped that sufficient references are included to enable the interested ecologist to obtain this information. Necessary definitions are provided and the general nature of systems considered. The relationship between systems analysis and problem solving is established, and the ecosystem is examined in the framework of the problem-solving processes. An example of a systems model is presented and the problems of investigating and manipulating systems and of organizing ecosystem descriptions are considered.

\footnotetext{
1 Received August 4, 1969; accepted October 1, 1969.

- Present address : Division of Plant Industry, CSIRO, Canberra, A.C.T., Australia.
}

\section{Systems Analysis, Systems, and ECOSYSTEMS}

\section{Systems analysis}

Systems analysis has rarely been defined when introduced into ecological studies. Watt (1968) suggests that it is the determination of those variables which are important in a system, and further adds that systems simulation, systems optimization, and systems measurement are other facets of the systems approach. Others, such as Priban (1968), view model building as the essence of the systems approach. Morton (1964) has suggested that systems analysis is no more nor less than scientific method itself, and that the distinguishing feature of the systems approach is the conscious application of scientific method to complex organizations in order that no important factor be overlooked, a view expressed by Pascal as "error comes from exclusion." These viewpoints are not necessarily mutually exclusive. Systems analysis is the application of scientific method to complex problems, and this application is further distinguished by the use of advanced mathematical and statistical techniques and by the use of computers. The computers are used as "number-crunching" calculating machines and as convenient tools for modelling systems too complex for analytic solutions to be presently possible. This modelling function is of great importance in studies of complex natural systems, for, provided the model can be treated as representing the real system for the purposes of the investigation, experiments can be performed on the model with a consequent gain in control and rapidity of response. A good model will obviously contain the important variables, so Watt's comments are pertinent. Equally, if natural systems are complex then the modelling phase of the systems analysis will be emphasized and Priban's emphasis accepted. Morton's more general view, since it includes both the others, seems the most acceptable since it does not presuppose 
some a priori emphasis on certain parts of the analysis.

\section{Systems}

A system is a collection of interacting entities, or alternatively it is a collection of parts, together with statements on the relationships, of some kind, between these parts. The interpretation to be given to the entities is the choice of the investigator, but the entities need not, and in general are not, in one-to-one correspondence with "real" things. They can represent classes of things, or classes of processes if this seems necessary. The state of the system at some point in space and time is described by the values of properties of the entities, and all properties used to so describe the system are termed endogenous. Variables which affect the interrelationships between entities, but which are not included in the state description, are called exogenous and form the environment in which the system acts. If endogenous properties are interchanged with other systems outside the defined one, then the system is said to be "open" for these properties. If there is no import or export, the system is closed. Representations of systems can take a variety of forms. Perhaps the commonest is as a network (Ford and Fullerson 1962, Harary, Norman, and Cartwright 1965) or as a matrix derived from such a network. An alternative mathematical representation is given by Rosen (1958), and an ecological example is the structural description diagrams of Dansereau, Buell, and Dagon (1966). Such general descriptions permit discussions about systems, but a computer program modelling a system is equally a representation of that particular system.

Any system is composed of subsystems defined for subsets of the entities. Each of these subsystems can be treated as a system in its own right, so that the definition of a system is recursive. ${ }^{3}$ An open system, that is, one open for at least one property, can be considered as a subsystem of some "higher" order system (Cooper 1969), and since each subsystem can be decomposed into subsubsystems, a hierarchy of systems is produced. A familiar example of such a hierarchy is . . . . -organism-organ-tissue-cell-organelle-. . . . . Obviously some means is required to prevent infinite regress, and in practical work this termination depends on the fidelity of the model of the system

${ }^{3}$ A simple example of recursive definition is the factorial of an integer number written $n !$. This can be calculated as follows:

$n !=n \times(n-1) \times(n-2) \times(n-3)$ $\times \ldots \ldots \times 3 \times 2 \times 1$

Equally the value can be calculated from the following rule.

$$
n !=\text { if } n=1 \text { then } 1 \text { else } n \times(n-1) !
$$

to the "real" system. This fidelity requirement will be discussed later.

Finally, it is necessary to define an ecosystem. An ecosystem is a system open for at least one property, in which at least one of the entities is classed as living. This definition is very broad, but restrictions imposed by ecologists to limit this definition for particular studies have not received much consideration. It must be remembered that an ecosystem is a special case of the general system and will possess all properties of the general system. Thus there is no restriction on the number of properties which may be used to describe the system, although many ecologists have so restricted themselves, with the consequent introduction of difficulties with an excessively large number of exogenous variables. There is certainly no restriction to studies of productivity or energy transfer, although many applications of systems analysis in ecology have been on these problems. Population models are systems models and so is the physiognomic description of vegetation. However, since the preponderance of systems studies in ecology have been studies of productivity, it will be convenient to phrase examples in these terms.

\section{Systems axd Problem Solving}

The recursive hierarchical nature of systems is closely paralleled in some theories of human problem solving (e.g., Simon and Newall 1962, Feigenbaum and Feldman 1963). Here an attempt is made to decompose insoltuble problems into subproblems. Any subproblems remaining insoluble are further decomposed, until hopefully all subproblems and their derivatives are soluble, when an attempt is made to reintegrate the solutions into a single solution of the original problem. The parallel between problem solving and systems can be drawn more closely, however. Ross (1967) distinguishes four phases in problem solving: (1) lexical, (2) parsing, (3) modelling, and (4) analysis (see also Morton 1964). In systems analysis these same four phases can also be identified: (1) delimination of the entities or parts; (2) the choice of relationships between entities which are of interest; (3) the specification of the mechanism by which these interrelationships take place; and (4) validation of the model of the system so produced and investigation of its properties.

Ross points out that the rules under which these phases are carried out must be agreed upon a priori, which is not a simple task. An obvious example of changing rules is given by the diversity of human language, which has an additional complication due to the possible existence of several 
scripts for one spoken language. Some of the phytosociological arguments on vegetation description appear to be arguments regarding rules of procedure, although the situation is complicated here because it is not clear that each system is intended to contain the same information. Ecologists must therefore agree on the rules to be used, otherwise comparison of systems will not be possible. Much of the difficulty lies in the choice of entities, and this will be discussed later.

The four phases of problem solving and systems analysis are used in the following sections as a framework in which to discuss problems in systems analysis.

\section{The lexical phase}

One of the most neglected problems in systems studies is the choice of the entities or parts which compose the system. It is commonly assumed that these are self-evident; yet the arguments which have taken place in areas such as the classification of organisms or vegetation concerning sampling, description, and measures of similarity suggest that this is not true. In taxonomy a hierarchy similar to the systems hierarchy is apparent-family, genus, species, etc., and taxonomists have agreed that while studies at any level are possible, the species level is in some way more important. It is by no means clear that the species level is a consistent level: the occurrence of "difficult" genera such as Hieracium, Rubus, or Quercus, and the varied degree of subtlety in characters used to describe and distinguish species in different families such as the Umbelliferae and the Magnoliaceae, attests some inconsistency. Yet the taxonomist has a distinct advantage over the ecologist in that there exists a generating system (Williams 1967), the genetic system, which constrains the possible variation, so that the lexical phase in taxonomy rests on the interpretation of genetic event patterns; that much of the genetic information available is not at the species level but within it is a practical problem though an unfortunate one. The ecologist has no such system presently available, and in the opinion of some ecologists there is no such system.

The choice of entities for the ecosystem is in part determined by the parsing phase, that is, by the nature of the relationships with which the system is concerned. The commonest choices have been between taxonomic, structural, and functional entities. Taxonomic is a convenient adjective to describe entities based on individual organisms, populations, and the commoner taxonomic categories of species, genera, and so on. Structural entities are based on life-form criteria, trees, shrubs, herbs, and bryoids providing a simple botanical example. Life-form criteria are in general more responsive to local environmental fluctuations than taxonomic criteria, since these latter employ characters selected to be invariant within taxa, wherever possible. Functional entities have perhaps received more attention in animal ecology, e.g., herbivore, carnivore, omnivore, although a variety of similar units exists in plant ecology, though less precisely defined, e.g., xerophyte, halophyte, and saprophyte. The definition of entities is not of course concerned with the ease of identification of these parts, although it may well be essential to provide common means of identifying the entities if different systems are to be compared. Of more consequence is the possibility of conversion from one set of entities to another. If one description employs structural categories and another taxonomic categories, how can the two be compared? Liriodendron tulipifera is a taxon which could certainly fall into the categories of shrub or tree, and on some definitions the seedlings would be classed as herbs. Even restriction to species as entities fails to resolve the problem, for this ignores all ecotypic and ontogenetic variation and the inconsistencies in the species level noted above.

It may be true that some ecosystems can only be compared at gross levels such as autotroph and heterotroph, for example marine and terrestrial systems. Yet because the United Kingdom has some 1,700 species of vascular plants, 900 bryoids, and various numbers of lichens and fungi, whereas Oak Ridge, Tennessee, has some 2,000 species of vascular plants alone, does not imply that comparisons of the two areas are only possible at some very gross level, even though the species complements are widely different. To demand that comparisons be possible with both very similar and very different ecosystems places severe restrictions on the possible choices of entities which can be employed to describe the systems. It may also be possible to describe systems in terms of a few simple ratios, such as the efficiencies which have been proposed, but this could equally reflect the wellknown half-truth that biologists, when given two numbers, divide one by the other.

Functional entities do not resolve the problems of choice any more than structural or taxonomic entities. Omnivores, for example, are both herbivorous and carnivorous, while insectiverous plants are both autotrophic and heterotrophic. Nonliving materials within the ecosystem are less well served with possibilities, while still having problems of ontogeny and chemical equilibrium, such as that between the various forms of nitrogen in the soil. Perhaps a distinction between solid, 
liquid, and gaseous phases is possible, but is this all?

Since the first phase of a systems analysis is the choice of entities, it is very necessary that an ecologist give considered thought to these problems. In a study of a single system, the problems may well be less acute than when comparison of systems is necessary. But the choice of entities is the ecologists' task and must not be given to the systems analysts by default.

\section{The parsing phase}

The second phase is concerned with the definition of the relationships between the selected entities. These relationships can be of any kind and need not be restricted to materials. It has been common practice in ecology to assume that the relationships are those relating to material which the system can reorder or reallocate among its parts. The relationships can, however, be spatial or temporal and need not concern materials at all. Such relationships are important in physiognomic description of vegetation. In view of the present great interest in productivity, however, attention will be concentrated on ecosystems models produced by such studies, and the properties relevant to them. These properties include energy, biomass, carbon, mineral nutrients, populations, individuals, water, and possibly information (in the form of genetic material). It does not include diversity in the sense in which this has been commonly used in ecology (Margalef 1947), which is a measure of the distribution of some property or properties over the entities, or some subset of them, in a single state description. Changes in diversity can provide useful indices of changes in this distribution caused by exogenous variables.

There is no restriction in the definition of the ecosystem given earlier on the use of several properties to describe the state of the system. The description can be multivariate. The importance of interaction between properties in such a multivariate system can be seen in work on mineral nutrient interactions and their effects on yield (Fig. 1). Such interactions are not of course limited to these particular variables. The difficulties of modelling and experimenting with multivariate systems do impose practical constraints on the investigator (Jacoby and Harrison 1962). The problem can be reduced to a univariate one by treating other properties as exogenous, although this increases the experimentation required. In comparing two systems which have been made univariate, it is essential to ensure that differences between systems do not become confounded with differences in treatment of the relocated exogenous variables.

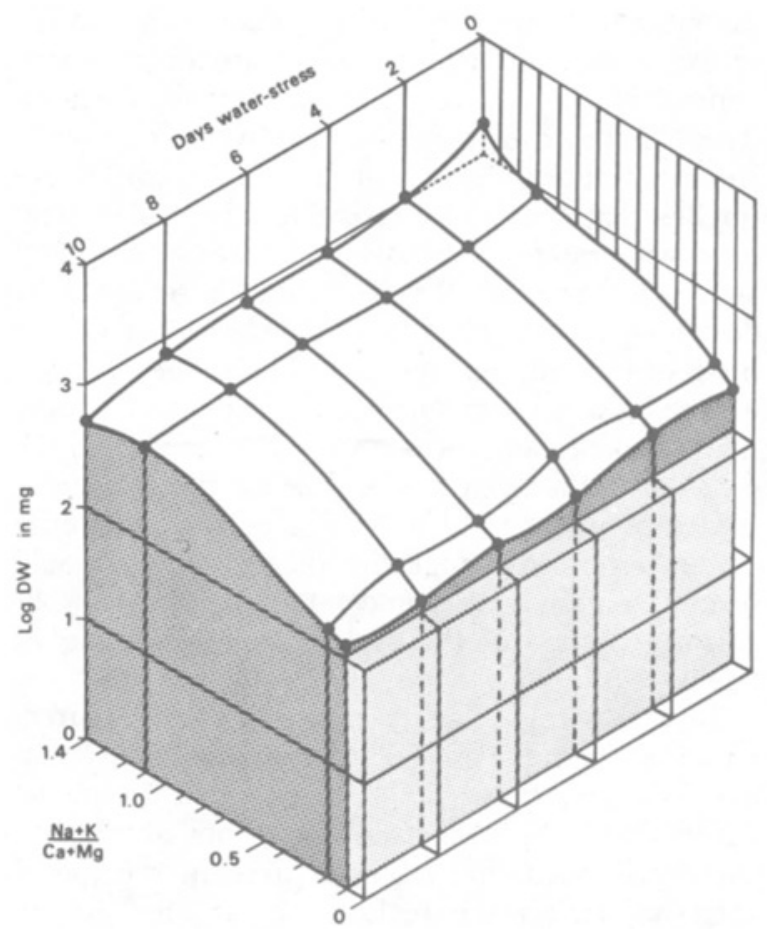

FIG. 1. Dry weight response of Atriplex inflata after 8 weeks in relation to days of withholding water and the ratio $(\mathrm{Na}+\mathrm{K}) /(\mathrm{Ca}+\mathrm{Mg})$.

In the context of the International Biological Program the property most favored has been energy fixed as carbon, usually as total biomass. This implies a preference against edible, palatable, or otherwise desirable biomass such as protein, which preference may not always be desirable. Thus wool growth in sheep may well be related to amounts of sulfur-containing protein rather than total biomass consumed. This is not to deny the importance of energy transfer as measured by total biomass, but merely to indicate that it alone will be but a partial representation of the "real" system and may not always be the most desirable. Other choices have been made including mineral nutrients, water, radioactive contaminants, and, with growing emphasis in the United States, pollutants in general. In all these cases, however, the possibility that a multivariate system might be more useful than the univariate one must be accepted and consideration given to the requirements of such a system model.

\section{Modelling}

Fidelity.-Having fixed the entities and the properties, the next phase is the specification of the mechanisms by which changes in the system, that is in the distribution of the properties across the entities, take place. In choosing these processes, an attempt is made to make the model of 
the system "mimic" the real system, either to increase understanding of the system or to attain control of the system over some range of states. This difference in possible objective is characterized by differing degrees of fidelity. High fidelity implies that the model resembles the real system for a wide range of states and changes in state and as a corollary, that this similitude is obtained by designing the model to follow presumed or known processes of the real system. The range of property values, for a given range of values of exogenous variables and for some entities, are called the outputs of the system, these being the particular values of interest. Knowledge of the sensitivity of the system to changes in some processes would be used to gain greater understanding of the mechanism of action of the system, and hence higher fidelity.

However, high fidelity is not always required. Provided that the model mimics the real system over some restricted range. that is, that the model outputs and the real system outputs are highly correlated, then the processes used in the model need not reflect the real system at all. As an example, in the description of spatial distributions of plants. several mathematical expressions may fit the data equally well, for example. Thomas' double poisson and the negative binomial distribution (Archibald 1948, Greig-Smith 1964). The mathematical expressions may well imply different models of the underlying real system, which may in fact agree with neither model, yet the results may be adequate. The simplest and most common ecosystem model is

\section{Input-Ecosystem-Output}

where the system itself is treated as a closed "black" box. This has been widely used in ecology (Van Dyne, Wright, and Dollar 1968), since it is the basis of univariate multiple regression. The attainment of high fidelity is expensive in time and in the effort required to obtain the precise and accurate data on which to build and validate the model. An analogy with sound reproduction is reasonably drawn. Telephone voice communication neither requires nor uses equipment necessary for the high-quality reproduction of music. ${ }^{4}$ The complexity of model required to attain high fidelity must be matched by the quality of the data. To continue the sound-reproduction analogy, a scratched recording is still scratched on the best equipment. The collection of adequate and rele-

\footnotetext{
- In statistics the problem of fidelity appears in the use of one distribution to provide an approximation to another. One example is the use of the normal distribution to approximate others such as the binomial, poisson, or Mann-Whitney U (see Siegel 1956).
}

vant data in studies of ecosystems will often be difficult if not impossible. For example, in studies of the interaction of radiant energy and a plant canopy, account must be taken of the spatial distribution of the stems and leaves. The collection of precise and detailed information on this feature is extremely difficult.

The processes to be defined must obviously depend on the choice of properties and entities. Changes in state of plant entities, for example, will require processes defining fixation of energy, carbon, nitrogen, and water, and other processes defining the reallocation of these properties among the entities. Some of the processes operate in sequence, the results of one forming an input to the next. They may also of course operate in parallel, that is, over the same time interval. This parallelism can be troublesome in some methods of investigating systems, such as simulation on digital computers which are essentially serial in operation. Special processing techniques (including special languages such as the SIMULA extension to ALGOL (Dahl and Nygaard 1966)) may be required.

The processes only change endogenous variables although they may employ both previous values of endogenous variables and exogenous variables in the calculation. It must also be realized that high fidelity in the definition of the processes does not guarantee high correlation between model and real outputs. The choice of exogenous variables also constrains the fidelity of the model. For example, consider two models of photosynthesis, one using mean day length to predict amount of carbon dioxide fixed, the other being more sophisticated and employing temperature, carbon dioxide, humidity, and radiation fluxes, together with data on spatial distribution of leaves to estimate the same value. The second might be expected to be of higher fidelity, yet by introducing appropriate stochastic variation into the first model it might be possible to make it of higher fidelity. This prediction requires less data, but the selection of the appropriate stochastic inputs would be troublesome. This emphasizes the importance of considering data-collection techniques when choosing the form of the processes (Watanabe and Abraham 1960). More than this, however, it reinforces the comment made earlier that the definition of the system in the ecologists' problem and is the result of interaction between available, or potentially available, information and the purposes for which the model is required. The modelling process may suggest areas where data-collection techniques might be improved so that a more faithful model becomes possible. One indication can be gleaned from economic models. The choice of 
interesting and practical models appears to be those models with 30-300 variables, with the experienced worker reducing the number (Forrester, personal communication).

Practical considerations. - Watt (1968) has presented a variety of approaches to the problem of defining processes; these by no means exhaust the possibilities. A very simple model of an ecosystem can be constructed consisting of four entities each of which represents a class and the properties and processes remain unspecified (Fig. 2). The same system can be presented in the form of a transition matrix, where each $a_{j k}$ represents the probability of transfer of a property between the $j^{\text {th }}$ and $k^{\text {th }}$ entity (Fig. 3). Similar matrices could be constructed for all properties, so that the entries can be interpreted as arrays of

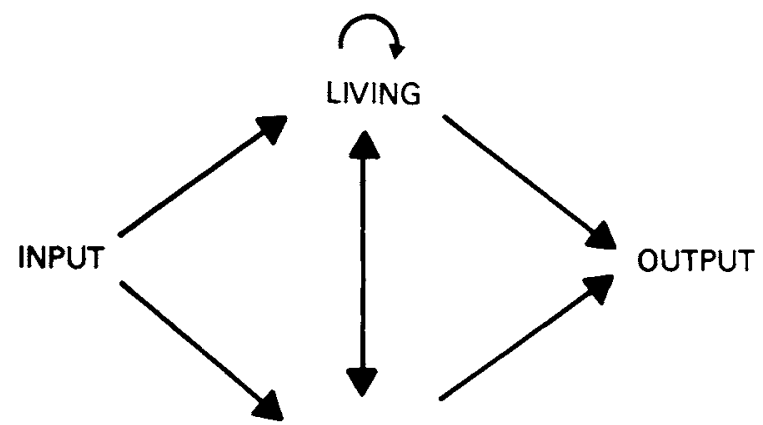

NONLIVING

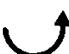

FIG. 2. The primitive model.

\begin{tabular}{|c|c|c|c|c|}
\hline & INPUT & LIVING & NONLIVING & OUTPUT \\
\hline INPUT & 0 & $a_{\mathrm{IL}}$ & $\mathbf{a}_{\mathbf{A} \mathbf{p}}$ & 0 \\
\hline LIVING & 0 & $a_{\text {IL }}$ & $a_{L p}$ & $a_{10}$ \\
\hline NONLIVING & ० & $a_{P L}$ & $a_{p p}$ & $a_{n}$ \\
\hline OUTPUT & 0 & 0 & 0 & 0 \\
\hline
\end{tabular}

FIG. 3. Transition matrix for the primitive model.

coefficients. To each of these there must be attached a corresponding process giving the next value of the $a_{j k}$ in terms of the present and previous values of the whole matrix and any additional exogenous variables. The nature of these functions is, of course, of great interest to ecologists. The model does not include some features of human information transfer where questions of the value, reliability, and credibility of information are involved. It would include demographic or population models which form the bulk of ecological work on systems (see Kerner 1957, 1959,
Whittle 1962, Bellman, Kagiwada, and Kalaba 1966, Garfinkel 1967a, b, Watt 1968, and others). Demographic models conveniently illustrate the duality between continuous and discrete models of systems. Many population models employ systems of differential equations which provide a continuous model of the system, including fractional values for the population total. But the population in most cases is discrete, being an integer number of individuals. Of the references given above only Whittle employs a discrete model (a discrete branching Markov process), probably because of the extra effort involved in the mathematics if restriction to integer solutions is imposed.

While demographic models can certainly be included in the ecosystem concept, it is also common to restrict the definition of ecosystem to models of the movement of materials or energy (e.g., Golley 1960, Olson 1963, Witherspoon, Auerbach, and Olson 1964, Patten 1965). If this restriction is accepted, then a slightly more complex model, as shown in Fig. 4, hopefully would improve in fidelity over the primitive model of Fig. 2. Autotroph in this model includes both energy and chemical fixation. Minor variants of this model in Fig. 5 show its generality.

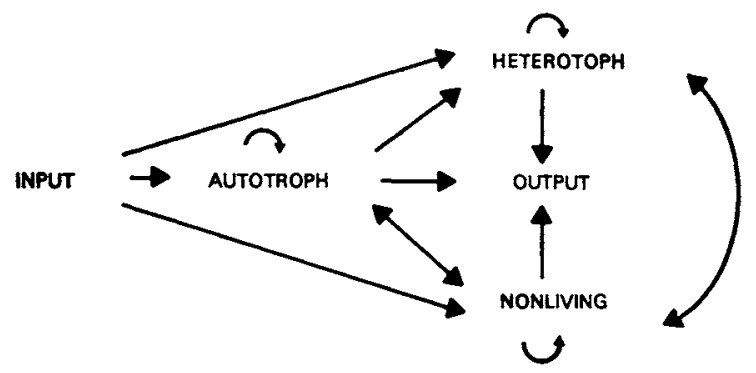

FIG. 4. Developments of the model for non-demographic systems.

While the network representations of Fig. 2, 4, and 5 are convenient visual models, mathematically the transition matrices corresponding to them are more easily handled. The meaning of such matrices can be considered geometrically. Consider a system with two entities and a single property. This can be represented as a point on a graph for any state. If the system changes due to change of an exogenous variable, then the point representing the system is displaced. A series of changes would trace a line, and the transition matrix contains the information describing this line. Of course in most models the graph is not in two dimensions, but the properties of the transition matrix still hold (see Keeney et al. 1967 for an extended description of this "state space" model).

The state of the system is a static description 
a. Perfect Autotroph Crop

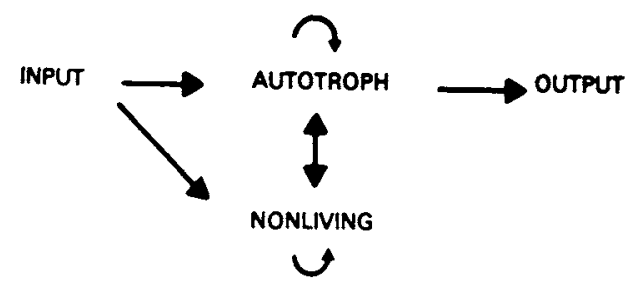

b. Cavernicolous or Hypogean System

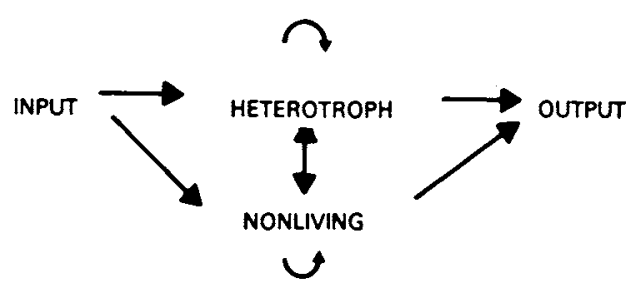

c. Isolated System or Spacecraft

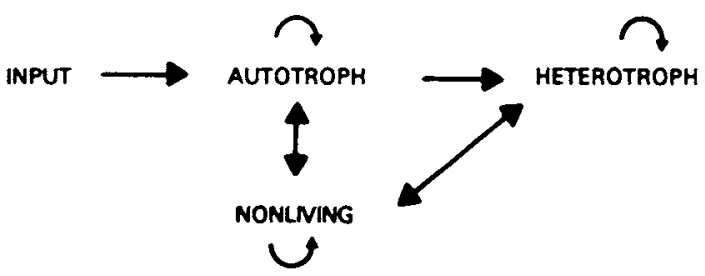

FIG. 5. Examples of restricted systems.

and the dynamics of changes in state are incorporated into the model by the processes. IVhile some systems may only show changes in response to changes in exogenous variables, in many systems and certainly in ecosystems the changes in state are partly determined by the previous states of the system, that is, by its history, by means of "feedback" or "memory." This is of course also included in the processes by making these employ previous values of the endogenous variables in the calculations. If these processes themselves employ parameters which change with time, the system is evolutionary, whereas if the parameters do not change with time, the process is stationary. The difficulties introduced by considering evolutionary processes are such that the majority of models employ stationary processes, although it is fairly clear that real ecosystems are strictly evolutionary. There is little work on the mathematics of evolutionary processes, and most of this is recent and at a somewhat adranced mathematical level. It would certainly be possible to permit evolutionary processes in simulation models, but this would involve a large increase in time and effort in an already time-consuming method, since the initial state of the system must be speci- fied very carefully for evolutionary processes. For many practical purposes over moderate time intervals the assumption of stationarity may be justified, although, as with multivariate models, the possibility of increasing fidelity by employing evolutionary models must be considered.

\section{Analysis}

The final stage of the systems approach is the analysis proper. This involves the solution of the model, in some sense, and the validation of the model outputs by comparing them to the real system outputs. In a few simple cases the model may be solved analytically using standard mathematical techniques. Models employing linear differential equations, for example, may be soluble, and in this case the sensitivity of the model to small changes in parameter values can also be calculated (Wilkins 1966). In general, however, no analytic solution will be available, and recourse must be made to the somewhat time-consuming simulation approach.

The likelihood that high fidelity will be desired suggests that stochastic models, incorporating random processes, will be preferable to deterministic models. This is due to the more realistic incorporation of variability in stochastic models and to the availability of estimates of the expected variability of the outputs. As an example of the greater realism of stochastic models, consider the spread of an infection through a population. Deterministic models suggest the existence of a critical population size at which there is a change from "no epidemic" to "epidemic" and the epidemic is of a fixed size. Stochastic models not only permit the epidemic to be variable in size, but also provide that, whatever the population size, there is an estimable chance of an epidemic occurring, and conversely of its not occurring (Bartlett 1960).

Simulation methods have been widely used both with analogue and with digital computers (Clymer and Graber 1964, International Business Machines Corporation 1966). Most of the ecological applications have used constant time increments, calculating the state of the model periodically. Less commonly event-orientated models have been attempted (Holling 1966). These essentially calculate the time interval between changes in state, so that periods when no change in state occurs require a constant computational effort independent of the length of the interval. Event orientation emphasizes the importance of recurrence intervals. which are ecologically important in determining survival times, where the event of "successful reproduction" and the event of "death" mark the intervals. Recurrence intervals are also 
important in migration and have been found useful in sampling vegetation (Williams, personal communication). Even if there are strongly periodic phenomena such as diurnal or annual cycles, an attempt to define events may force the modeller to consider his system in greater detail.

After a model of the system has been established and some means of investigating its responses has been provided, the crucial problem of the validation of the model remains. Validation may involve the functional form of the processes and the parameters supplied as constants to these processes, but primarily the interest lies in how well the model outputs mimic those of the real system, that is, in the fidelity of the model over the range of interest. If high fidelity is required there will usually be a process of successive approximation, with the model being progressively altered until the desired fidelity is obtained. This requires, of course, some measure of fidelity to assess the disparity between model and reality. Since the processes usually involve subsystems of the model, validating the processes is essentially also a process of measuring the fidelity of a system, in that the outputs of the subsystems to the complete system should presumably also be of high fidelity.

The difficulty of validating outputs depends on the features which it is desired to mimic. If only mean values must be estimated, the disparity can be measured by a test akin to Students' $t$, and various techniques are available to increase the precision of the comparison, mostly developed in Monte-Carlo studies (Hammersley and Morton 1956). These include such methods as Russian roulette, antithetic variables, and regression. If, however, the variance of the outputs or features of the transient response of outputs to particular changes in exogenous variables is required, the problem is more complex. The outputs form a correlated series of observations, and the comparison and investigation of such series present considerable statistical problems (see Quenouille 1957, Robinson 1967, Jenkins and Watt 1968). Rarely do ecological models specify which features of the output are to be reproduced by the model. Watt (1961) has provided examples of functions which produce outputs of given forms, and the use of least-squares surface fitting can also aid in the selection of possible functions to provide specific output forms. Perhaps the most general techniques are those of Wiener (1949), though these require a large amount of data.

Validation of the parameters of the processes involves searching the response surface of the model to ohtain "best" estimates. A variety of techniques might be of use here, including those due to Hooke and Jeeves (1961), Spang (1962), and
Narquardt (1963). The general problem of estimation in simulation studies has received most attention in engineering and management studies (e.g., Burdick and Naylor 1966, Fishman 1967, Fishman and Kiviat 1967), but it must be remembered that even if the model is validated, extrapolation beyond the limits of such validation is the responsibility of the ecologist. It would be foolish to say that such extrapolation is never justified, but the justification is not mathematical or statistical.

\section{An Autotroph System}

A diagrammatic representation of a precipitation-evapotranspiration ( $\mathrm{PET}$ ) system is used as an example of the models employed in systems analysis (Fig. 6). This is not the only model of this system since both Crawford and I.insley (1966) and Hufschmidt and Fiering (1966) incorporate simple expressions in their larger models to represent the whole of the PET system. Equally, more detailed models might be built up from the equations describing the transfer of heat and water vapor between leaf and atmosphere, and corresponding detailed study of the distribution of stem and leaves. The fidelity of the model will depend on the specifications of the processes by which the transfers of the property, here water, between entities is to be made. Such specifications have been provided and the resulting model converted to a computer program. The diagram shows only the connections between entities which were considered in the modelling.

Two subsystems are easily identified, one modelling the entry of water into the soil, the other modelling its return to the atmosphere. These interact at two points, since changes in the amount of leaves (and their distribution) will affect interception. and changes in the amount of roots will affect water extraction from the soil by the plant. Conversely, both leaf and root growth will be dependent on water availability.

Three other systems are explicitly included. The photosynthetic system requires inputs from the leaves and will feedback to both leaves and roots. This feedback is accomplished by the growth system which is responsible for the partition of photosynthate between the parts of the plant. The atmospheric system provides the source of water and the radiation which finally controls the loss of water. A fourth system could easily be added to introduce the effects of topography on input and output of water as runoff to or from other areas, and possibly erosion effects of such runoff. All the systems here operate in parallel in that they all operate simultaneously.

An equal time model of this system would re- 


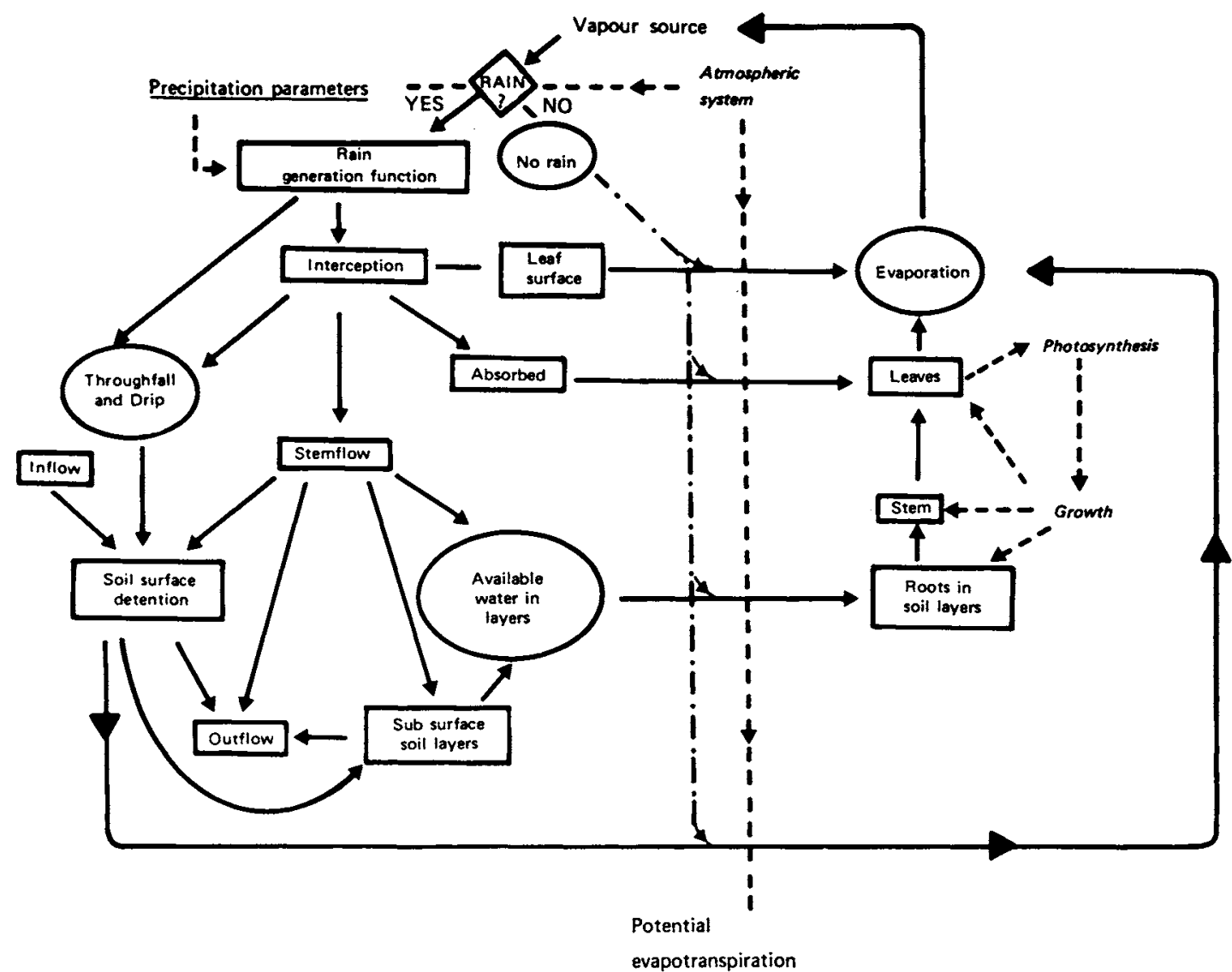

KEY
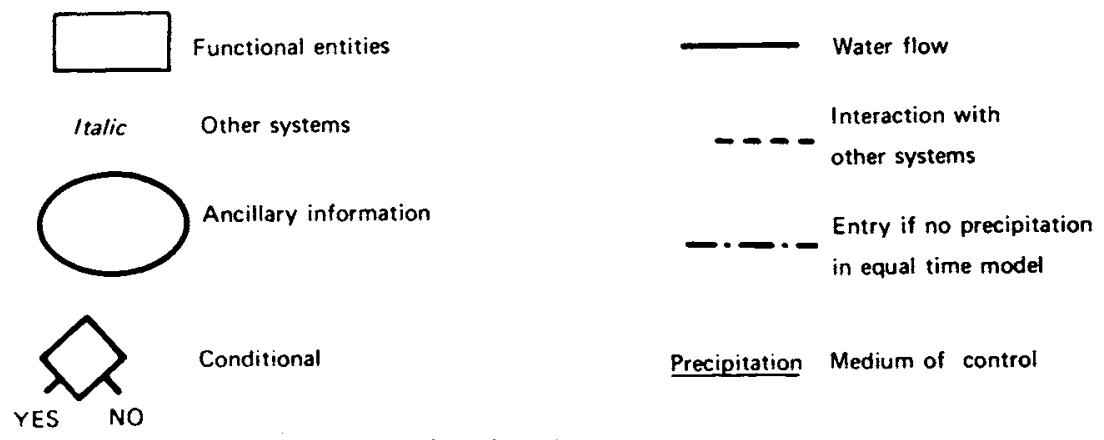

FIG. 6. The PET system.

view the description of the entities, which in this case would be the water contents and size of plant parts, at periodic intervals recalculating when necessary. During these periods the state is assumed to be constant, but the interval can be arbitrarily small at the expense of more computation. Given the periodicity of instrumental recording this might be acceptable, but the existence of continuous recorders permits the event-orientated model to be investigated.

In the event-orientated model time is variable, but during the interval between events the pro- cesses are assumed to proceed in a determinate manner. The events here would mostly be effects on the rates of water movement and on the growth rates. The systems and subsystems need not operate synchronously, each having its own event timing. Thus the photosynthetic system would show no events during darkness, and the growth system might show seasonal and ontogenetic effects. Dahl and Nygaard (1966) present a simple event model of an epidemic which illustrates the computer-programming techniques required. The event technique operates as if it followed small 
packages of water through the system and in this way transforms the parallel operation of the systems to a sequential operation. The choice between the two models, event or periodic time, will finally depend on the information available and the user's preferences. The PET system has in fact been programmed in both periodic and eventtime forms (Cooper, personal communication), with the latter proving computationally more effcient.

With either approach to modelling the models may be deterministic or stochastic. The stochastic model effectively replaces certain constants in the deterministic model with random variables drawn from appropriate statistical distributions. This drawing need not in fact be strictly random since by careful manipulation of the technique of drawing, the precisions of comparisons between the performance of the model under varying conditions may be increased. The technique is related to stratified sampling. While the appropriate distributions to use are relatively specific to individual problems and, indeed, form one of the most difficult parts of model building, for very rare events it may be possible to make use of the fact that extreme values have only three possible distributions (Gumbel 1958). This might appear a marginal advantage as there remains some choice to be made. However, this particular problem is ustrally solved by an automatic choice of the exponential distribution probably because of its ease of computation, and without regard for the alternatives. The systems analyst may indeed accept the simple exponential distribution, but he should be aware of the alternatives.

One of the interesting possibilities of controlling the "random" numbers depends on the nonexistence of such numbers. Random numbers are, in fact, pseudorandom in that they pass some of the tests of randomness which are possible, but not all. The infinite number of such tests makes it impossible to know if any set of numbers is random. Provided that the numbers are random for the tests employed, nonrandomness can be incorporated to reduce the effects of unimportant sources of variation. Tocher (1963) considers the possibilities in some detail.

\section{Fxperimentation Control and Optimization}

The techniques and considerations of the previous sections will hopefully lead to a valid model of the ecosystem. We will now consider means of using such a model of a single system as a guide to the management of the real system. This will involve experimenting with the real and model systems, identifying the parameters of the system which will enable it to be controlled, choosing a value function by which the performance of the system is to be measured, selecting the route to some desired state, and maintaining the system at or near this desired point. The advantages of using the model system lie in the ease and rapidity with which experiments may be carried out, and the possibility of including experiments which might be totally destructive in the real system. The disadvantages lie in the restricted range of confirmed validity of the model and in its fidelity even within this range to the real system which it is desired to control.

Designing experiments for model systems will necessarily involve use of the techniques developed in statistics for efficient experimenting, though often in an unusual form. The output of the experiments forms a response surface, and special experimental designs have been developed for studying there (Box and Draper 1959, Cochran 1963, Draper and Lawrence 1965). Since the response is a correlated sequence of values, the "growth curve" techniques of Roa (1965) and Potthof and Roy (1964) may be of assistance (see also Whittle 1963, Phattaford 1965, Spent 1967). The response may of course be multivariate, and correspondingly so must the methods of analysis (see e.g. Seal 1964), and may equally involve relaxation of assumptions of normality thus necessitating the use of non-parametric methods (Box and Watson 1962, Mood and Graybill 1963, Tiku 1964). Because the experiments are carried out on the model, efficiency and precision may be improved by restrictions imposed on the model which could not be imposed on the real system. One technique would be restrictions on the choice of "random" numbers as mentioned in the previous section, such as repeating the same sequence of "random" numbers both in the control and the experimental model solutions. A computer aid design may also be used in some cases (Kennard and Stone 1969).

The experiments lead towards control of the system, enabling a manager to manipulate the system towards some point and to maintain the system in the neighborhood of this point. Almost always there will be constraints on the actions available to the manager, such as avoiding certain states, restrictions on materials, and so on. General mathematical control principles are known (e.g., Pontryagin's continuous maximization principle, for which see Fan 1966), but these have proved difficult to apply in practice. Control implies the existence of a desired state and some means of assessing the importance and hence the size of any deviation from this state. Related to this is the need to measure the effects of any control operation. These subjects have been 
studied in detail in operations research (Bellman 1961, Muhzam 1963, Box and Tiao 1965). Maintenance of the system near the desired point means the control of variations in the outputs of the system. For example, management of a watershed to have flow proportional to demand would be ideal for hydroelectric power generation, where overproduction is worth little and underproduction is extremely costly. The quest for high fidelity models seems to result from the detailed control necessary, coupled with an assumption that the control of endogenous variables will be more selective, more efficient, and less expensive than control of exogenous variables. The truth of this assumption is debatable since the cost of obtaining the required detail in the model must also be considered. Both endogenous and exogenous variables can be manipulated in many systems.

Efficient control will usually imply the selection of important variables for which several techniques have been developed. Sensitivity analysis (Radanovic 1966, Wilkins 1966) is widely used to study the effects of small perturbations where the effects can be assumed to be nearly linear. Other approaches for isolating important variables exist, such as stepwise multiple regression, canonical correlation analysis (Kendall 1957), multiple predictive analysis, and two-parameter numerical taxonomy (Macnaughton-Smith 1965) or factor analysis (Lawley 1940, Harman 1966). Box and Jenkins (1962) have considered some statistical aspects of control.

The development of optimal control policies is one portion of the overall control process. The goal is provided by a "supersystem," and in this case the individual optimality of subsystems does not ensure the optimality of the system as a whole. An ecosystem might be evaluated in turn (in an appropriately organized society) by an administrative system, a political system, and a social system which employs a judicial system to enforce its control measures (Price 1965, Bulkley and McLaughlan 1966). The goals of all these evaluting systems must be defined and may often be conflicting. For the ecologist it is an evaluating function which is required rather than the goal itself, and this function may constrain the operation of the model and the operations of the managers. The existence of such an evaluating function is crucial, but two further problems are also apparent.

The first of these concerns the existence of "local" optima, which makes the search for the overall optimum more complex. Methods such as linear programming and its extensions to integer, quadratic, stochastic, and dynamic programming (Bellman 1957, Churchman, Ackoff, and Arnoff
1961, Wolfe 1962, Dantzig 1963, Watt 1963) have proved useful initial guides even if the system models do not always precisely fit the mathematical specifications (see Serck-Hanssen 1963, Watt 1963, Petrini 1964, Heady and Egbert 1964). Other workers have used statistical decision theory in efforts to determine optimal policies for action (e.g., Dillon and Heady 1960, Findler 1966).

The second problem is that of moving from the present state to the optimal one. Here the techniques of network analysis as planning aids are useful, including critical path analysis, resource allocation scheduling, program evaluation, and review techniques and transportation methods (Hein 1967. Haase 1960, Davis 1965, Martino 1965, Davis 1968). Since the majority of these methods require computer assistance with the calculation and the systems model itself will often be in the form of a computer program, it is interesting to speculate on what additional information is required to enable the computer to design its own experiments, and after analysis to report both the optimal point and the method of reaching it. Certainly cost functions, value functions, and constraints are required, but whether this is sufficient information is not known. As yet the evaluation of ecosystems is at a fairly gross level, and the ecologist is educating himself and others in the extent and degree of complexity inherent in ecosystem management, while avoiding the grosser catastrol hes.

\section{Comparison and Organization of Ecosystems}

Some of the problems of comparing ecosystems will be considered briefly. Such comparisons are desirable partly because of the spatial and temporal variation between systems, and partly because as a "pure" science ecology will include the study of patterns in ecosystems.

While it would be possible to extend the description of a system to include those with which it interacts, this will often be impracticable. The pattern of ecosystems with respect to environmental factor, and the processes of successional change are both areas where the comparison of ecosystems is desirable. Such comparisons have for the most part been made by comparing the diversity of the systems as measured by a single property. In regetation studies this has commonly meant comparison of species lists. The emphasis placed on functional entities by Lindeman (1942) and the increasing use of indicator species has not replaced the taxonomic comparisons, and the success of floristic miethods such as those of Heikurainen (1964) suggests that there is strong relationship between functional and taxonomic classes. For some purposes it may be necessary to reconsider 
presently unfashionable entities, such as the synusiae of Lipmaa (1939).

The process of comparison and the organization of the resulting information to exhibit the patterns of ecosystem structuring is itself a systems process. Clowes (1967), in discussing similar problems in the computer processing of pictures, again distinguishes the four phases: the definition of parts, the provision of a grammar of parts (parsing phase), the representation of this part in relationship to structure in the machine (modelling), and the final analysis of the picture representation (analysis phase). A formal process of comparing ecosystems will itself involve these four phases, although the last analytic phase will be some numerical organization method such as classification, ordination, or spectral analysis (Robinson 1967, Jenkins and Watt 1968). The ecological difficulties all lie in the selection of the entities or parts and the selection of the relationships between the parts which are of interest in the particular study. Simple examples of relationships important in some areas of ecology are the concept of "epiphyte," which involves the relationship "growing on," and the concept of stratification of vegetation involving the spatial relationships "above" and "below." Selection of the appropriate relationships from the many available is a major ecological problem.

While the processing of the ecosystem description is possible, this is not the place to discuss the means available to represent ecosystems and the techniques necessary to compare the complex structures. The majority of the problems so far encountered in this area have been solved, in the sense that something can be done. although the ecological implications of the available solutions is not always clear.

\section{Concluston}

The questions to be asked of this brief account of systems analysis fall into three categories. First, what additional knowledge must the ecologist acquire before he can use systems methods? Second, what ecological questions must be answered before he can apply the methods? Third, what can he hope to gain by using such methods? These will be consiclered in order.

It is apparent that systems analysis includes a wicle variety of mathematical and statistical techniques and borders many areas, including computation, picture processing, language processing, and problem solving. The ecologist need not be fluent in all these areas, but some means of communicating between them seems desirable. The methods used in systems analysis are rarely phrased in ecological language, and the ecologist will certainly have to phrase his questions in nonecological terms if the developers of the methods are to assist him.

The ecological questions rest on the need for this translation, for the ecologist defines the problems in which he is interested and must interpret them to the assisting workers. For systems he must specify the parts and the relationships and be prepared to modify these definitions in the light of data-collection problems and the fidelity requirement. If he is attempting to control or modify an ecosystem in the light of his models, he must have the desired objective stated, some means of evaluating departures from this state, and some idea of the external constraints imposed on the system and its managers. As an example where the objective function has been variously interpreted, consider the problem of controlling fire in forests. Australian foresters are at present recommending frequent controlled burning as a means of reducing fire hazards. Such a solution has one disadvantage, i.e., the frequency of burning increases due to the selection of rapidly recovering and fire-tolerant species. It also ignores the problems raised by loss of nutrients due to burning and the effects of such losses on the productivity of the trees, since the environment is already nutrient poor. As a solution to the problem of reducing fire risks inmediately, controlled burning is probably acceptable, but this is in fact only part of the system.

The gains to be expected from a systems approach come from the precise statement of the problems and the discipline imposed by an ordered approach to the complexities of the real system. It is unlikely that an optimal solution to any problem will be attained directly, a process of successive approximation being likely. That the discipline is helpful can be seen from experience with one technique of management, the program evaluation and review technirue (PERT, see Davis 1968). This technique has been credited with saving large sums of money, yet on closer inspection the method consists of little more than an explicit statement of what goes on and in what order! It should also be clear, however, that systems analysis is not a panacea, and its use will involve the ecologist in extending his knowledge, biological and other, before gaining much reward. Hopefully the use of systems methods will prevent ecologists from joining those "who saw the effect but not the cause."5

\section{ACKNOHLEDMENTS}

It is a great pleasure to acknowledge the advice and aid of Professor C. F. Cooper under whose auspices I worked at the University of Michigan as a participant in a re-

${ }^{5}$ St. Augustine Contra Pelagium IV 60. 
search project sponsored by Cooperative Research, U.S. Department of Agriculture. It is also a pleasure to record my thanks to Dr. J. Olson of Oak Ridge National Laboratory and other members of the discussion group on systems analysis. My thanks to Professor Cooper again for permission to use the PET model and to Dr. D. J. Anderson of the Australian National University for the response surface diagram.

\section{Literature Cited}

Archibald, E. E. A. 1948. Plant populations. I. A new application Neyman's contagious distribution. Ann. Bot. (London) N.S. 12: 221-235.

Bartlett, M. S. 1960 . Stochastic population models in ecology and epidemiology. Methuen, London. 90 p.

Bellman, R. 1957. Dynamic programming. Princeton Univ. Press, Princeton, N.J. 342 p.

1961. Adaptive control processes: a guided tour. Princeton Univ. Press, Princeton, N.J. 255 p.

Bellman, R., H. Kagiwada, and R. Kalaba. 1966. Inverse problems in biology. J. Theor. Biol. 11: 164 167.

Box, G. E. P., and N. R. Draper. 1959. A basis for the selection of a response surface design. J. Amer. Statist. Ass. 54: 622-654.

Box, G. E. P., and G. M. Jenkins. 1962. Some statistical aspects of adaptive optimisation and control. J. Roy. Statist. Soc., Ser. B, 24: 297-343.

Box, G. E. P., and G. C. Tiao. 1965. A change in level of a nonstationary time series. Biometrika 52: 181192.

Box, G. E. P., and G. S. Watson. 1968. Robustness to non-normality of regression tests. Biometrika 49: 93-106.

Bulkley, J. W., and R. T. McLaughlin. 1966. Simulation of political interaction in multiple purpose river basin development. Mass. Inst. Technol., Dep. Civil Eng. Hydrodyn. Lab. Rep. 100. 307 p.

Burdick, D. S., and T. H. Naylor. 1966. Design of computer simulation experiments for industrial systems. Commun. Ass. Comput. Mach. 9: 329-339.

Bush, R. R., and F. Mosteller. 1955. Stochastic models for learning. John Wiley and Sons, Inc., New York. $365 \mathrm{p}$.

Churchman, C. W., R. L. Ackoff, and E. L. Arnoff. 1961. Introduction to operations research. John Wiley and Sons, Inc., New York. 645 p.

Clowes, M. B. 1967. Perception, picture processing and computers, p. 181-198. In N. L. Collins and D. Mitchie [ed.] Machine intelligence I. Oliver and Boyd, Edinburgh \& London.

Clymer, A. B., and G. F. Graber. 1964. Trends in the development and applications of analog simulations in biomedical systems. Simulation 4: 41-58.

Cochran, W. G. 1963. Sampling techniques. John Wiley and Sons, Inc., New York. 330 p.

Cooper, C. F. 1969. Ecosystem models in watershed management, p. 309-324. In G. M. Van Dyne [ed.] The ecosystem concept in natural resource management. Academic Press, New York.

Crawford, N, and R. Linsley. 1966. Digital simulation in hydrology. Stanford Watershed Model IV. Stanford Univ., Dept. Civil Eng. Tech. Rep. 39. 210 p.

Dahl, O. J, and K. Nygaard. 1966. SIMULA: an ALGOL-based simulation language. Commun. Ass. Comput. Mach. 9: 671-678.

Dansereau, P., P. F. Buell, and R. Dagon. 1966. A universal system for recording vegetation. Sarracenia 10: 1-64.
Dantzig, G. S. 1963. Linear programming and extensions. Princeton Univ. Press, Princeton, N.J. 625 p.

Davis, E. W. 1965. Resource allocation in project network models : a survey. J. Ind. Eng. 14: 177-188.

Davis, J. B. 1968. Why not PERT your next resource management problem. J. Forest. 66: 405-408.

Dillon, J. L., and E. O. Heady. 1960. Theories of choice in relation to farmer decision. Iowa State Univ. Agr. Exp. Sta. Res. Bull. 485. 23 p.

Draper, N. R., and W. E. Lawrence. 1965. Designs which minimise model inaccuracies : cuboidal regions of interest. Biometrika 52: 111-118.

Fan, Liang-Tseng. 1966. The continuous maximum principle. John Wiley and Sons, Inc., New York. $411 \mathrm{p}$.

Feigenbaum, E. A., and J. Feldman. 1963. Computers and thought. McGraw-Hill Book Co., Inc., New York. $535 \mathrm{p}$.

Findler, N. V. 1966. Human decision-making under uncertainty and risk: computer based experiments and a heuristic simulation program. Proc. A. F.I. P.S. 1965 Fall Joint Computer Conf., Pt. 1: 737-752.

Fishman, G. S. 1967. Problems in the statistical anal$y$ sis of simulation experiments: the comparison of means and the length of sample records. Commun. Ass. Comput. Mach. 10: 94-99.

Fishman, G. S., and P. J. Kiviat. 1967. The analysis of simulation generated time series. Manag. Sci. 13: $525-557$.

Ford, L. R., Jr, and D. R. Fulkerson. 1962. Flows in networks. Princeton Univ. Press, Princeton, N.J. $194 \mathrm{p}$.

Garfinkel, D. A. 1967a. A simulation study of the effects on simple ecological systems of making rate of increase of population density dependent. J. Theor. Biol. 14: 46-58.

—— 1967b. Effect on stability of Lotka-Volterra ecological systems of imposing strict territorial limits on populations. J. Theor. Biol. 14: 325-327.

Glanzer, M., and R. Glaser. 1959. Techniques for the study of group structure and behaviour. 1. Analysis of structure. Psychol. Bull. 56: 317-332.

Golley, F. B. 1960. Energy dynamics of a food chain of an old field community. Ecol. Monogr. 30: 187206.

Greig-Smith, P. 1964. Quantitative plant ecology. Butterworth, London. 198 p.

Gumbel, E. L. 1958. Statistics of extremes. Columbia Univ. Press, New York. 375 p.

Halmos, P. R., and H. E. Vaughan. 1950. The marriage problem. Amer. J. Math. 72: 214-215.

Hammersely, J. M., and K. W. Morton. 1956. A new Monte Carlo technique: antithetic variables. Proc. Camb. Phil. Soc. 52: 449-475.

Harary, F., and P. Lipstein. 1962. The dynamics of brand loyalty: a Markovian approach. Oper. Res. 10: $19-40$.

Harary, F., R. Z. Norman, and D. Cartwright. 1965. Structural models: An introduction to the theory of directed graphs. John Wiley and Sons, Inc., New York. $415 \mathrm{p}$.

Harman, H. H. 1966. Modern factor analysis. 2nd ed. Univ. Chicago Press, Chicago. 474 p.

Hasse, M. 1960 . Uber die Behandlung Graphen theoretischer Probleme unter Verwendung der Matrizenrechnung. Wiss. Z. Tech. Univ. Dresden 10: 13131316.

Heady, E. O, and A. C. Egbert. 1964. Regional programming of efficient agricultural patterns. Econometrika 32: 374-386. 
Heikurainen, I. 1964. Suptyyppien Ojituskelpoisus : metsänkasvatusta silmälläpitäen. Kirjayhytyma, Helsinki.

Hein, L. W. 1967. The quantitative approach to managerial decision. Prentice Hall, New York. 386 p.

Holling, C. S. 1966. The functional response of invertebrate predators to prey density. Mem. Ent. Soc. Can. 48: 1-85.

Hooke, R., and T. A. Jeeves. 1961. Direct search solutions of numerical statistical problems. J. Ass. Comput. Mach. 8: 212-229.

Hufschmidt, M. M., and M. B. Fiering. 1966. Simulation techniques for design of water resource systems. Harvard Univ. Press, Cambridge, Mass. 212 p.

International, Business Machines Corporation. 1966. Bibliography on simulation. Report 320-0926-0. White Plains, N.Y.

Jacoby, J. E., and S. Harrison. 1962. Multivariable experimentation and simulation models. Naval Res. Log. Quart. 9: 121-136.

Jenkins, G. M., and D. G. Watt. 1968. Spectral analysis and its applications. Holden Day, San Francisco, Calif. 525 p.

Keeney, M. G., H. E. Koenig, and R. Zemach. 1967. State space models of educational institutions. Michigan State Univ. Div. of Engineering Research. East Lansing, Mich. 48 p.

Kendall, M. G. 1957. A course in multivariate analysis. Griffin, London. 185 p.

Kennard, R. W., and L. A. Stone. 1969. Computer aided design of experiments. Technometrics 11: 137148.

Kerner, E. H. 1957. A statistical mechanics of interacting biological species. Bull. Math. Biophys. 19: 121-146.

- 1959. Further considerations on the statistical mechanics of biological association. Bull. Math. Biophys. 21: 217-255.

Lawley, D. N. 1940. The estimation of factor loadings by the method of maximum likelihood. Proc. Roy. Soc. Edinb., a, 60: 64-82.

Lindeman, R. L. 1942. The trophic dynamic aspect of ecology. Ecology 23: 399-418.

Lippmaa, T. 1939. The unistratal concept of plant communities. Amer. Midland Natur. 21: 111-145.

Macnaughton-Smith, P. 1965 . Some statistical and other techniques for classifying individuals. Home Office Res. Unit Rep. 6. H.M.S.O., London. 33 p.

Margalef, D. R. 1947. Information theory in ecology. Mems. R. Acad. Barcelona 23: 373-440. (Trans. in Gen. Systems 3: 36-71. 1958).

Marquardt, D. W. 1963. An algorithm for least squares estimation of nonlinear parameters. J. Soc. Ind. Appl. Math. 11: 431-441.

Martino, R. L. 1965. Advances in network techniques: an introduction to MAP. Data Process. 8: 231-257.

Mood, A. M., and F. A. Graybill. 1963. Introduction to the theory of statistics. McGraw-Hill Book Co. Inc., New York. 443 p.

Morton, J. A. 1964. From research to industry. Int. Sci. Technol., May 1964: 82-92, 105.

Muhzam, H. 1963. On multivariate trends. Paper presented to the 5th Int. Biometric Conf., Cambridge, England.

Olson, J. S. 1963. Energy storage and the balance of producers and decomposers. Ecology 44: 322-331.
Orcutt, G. H. 1960. Simulation of economic systems. Amer. Econ. Rev. 50: 893-907.

Patten, B. C. 1965. Community organization and energy relationships in plankton. Oak Ridge Nat. Lab. Rep. ORNL-3634.

Petrini, P. 1964. Competition between agriculture and forestry under Swedish conditions. Lantbrukshögskolansannalar 30: $156 \mathrm{p}$.

Phattaford, R. M. 1965. Sequential analysis of dependent observations. Biometrika 52: 157-165.

Potthof, R. F, and S. N. Roy. 1964. A generalized multivariate analysis of variance model useful especially for growth curve problems. Biometrika 51: 313-326.

Priban, I. P. 1968. Forecasting failure of health. Sci. Cult. 34: 232-235.

Price, D. K. 1965 . The scientific estate. Harvard Univ. Press, Cambridge, Mass. $321 \mathrm{p}$.

Quenouille, M. H. 1957. Analysis of multiple time series. Griffin, London. $105 \mathrm{p}$.

Radanovic, L. [ed.] 1966. Sensitivity methods in control theory. Proc. Int. Symp. Dubrovnik, Yugoslavia. Pergamon Press, New York. 456 p.

Rao, C. R. 1965. Theory of least squares when the parameters are stochastic and its application to the analysis of growth curves. Biometrika 52: 447-458.

Robinson, E. A. 1967. Multichannel time series analysis. Holden Day, San Francisco, Calif. 298 p.

Rosen, R. 1958. The representation of biological systems from the standpoint of the theory of categories. Bull. Math. Biophys. 20: 317-341.

Ross, D. T. 1967. The AED approach to generalized computer-aided design. Proc. Ass. Comput. Mach. National Meeting 1967 : 367-385.

Seal, H. L. 1964. Multivariate statistical analysis for biologists. Methuen, London. 209 p.

Serck-Hanssen, J. 1963. A programming model for a fishing region in northern Norway. Regional Science Association Papers 12: 107-118. Lund Congress.

Siegel, S. 1956. Nonparametric statistics for the behavioural scientist. John Wiley and Sons, Inc., New York. 201 p.

Simon, H. A., and A. Newall. 1962. Simulation of human thinking, p. 95-131. In M. Greenberger [ed.] Computers and the world of the future. Mass. Inst. Technol. Press, Cambridge, Mass.

Spang, H. A. 1962. Review of minimization techniques for non-linear functions. Soc. Ind. Appl. Math. Rev. 4: 363-365.

Spent, P. 1967. Estimation of mean growth curves. J. Theor. Biol. 17: 159-173.

Tiku, M. L. 1964. Approximating the general nonnormal variance ratio sampling distribution. Biometrika 51: 83-95.

Tocher, K. D. 1963. The art of simulation. English Universities Press, London. 184 p.

Van Dyne, G. M., R. G. Wright, and J. F. Dollar. 1968. Influence of site factors on vegetation productivity. ORNL-TM 1974 Contract No. W-7405-eng26. Oak Ridge National Laboratory. 238 p.

Watanabe, S., and C. T. Abraham. 1960. Loss and recovery of information by coarse observation of stochastic chain. Information and Control 3: 248-278.

Watt, K. E. F. 1961. Mathematical models for use in insect pest control. Can. Entomol. Suppl. 19: 1-62.

—_. 1963. Dynamic programming, 'Look-Ahead' programming and the strategy of insect pest control. Can. Entomol. 95 : 525-536. 
1968. Ecology and resource management: a quantitative approach. McGraw-Hill Book Co., Inc., New York. 450 p.

Whittle, P. 1962. Topographic correlation, power-law covariance functions and diffusion. Biometrika 49: 305-312.

1963. Prediction and regulation. English Universities Press, London. $147 \mathrm{p}$.

Wiener, N. 1962. The extrapolation, interpolation and smoothing of stationary time series. Mass. Inst. Technol. Press, Cambridge, Mass. 163 p.
Wilkins, R. D. 1966 . General time varying systems error sensitivity analysis. Commun. Ass. Comput. Mach. 9: 855-859.

Williams, W. T. 1967. Numbers, taxonomy and judgement. Bot. Rev. 33: 379-386.

Witherspoon, J. P., S. I. Auerbach, and J. S. Olson. 1964. Cycling of caesium-134 in white oak trees. Ecol. Monogr. 34: 403-420.

Wolfe, P. 1962. Recent developments in non-linear programming, p. 156-187. In F. L. Alt and M. Ruhinoff [ed.] Advances in computers 3. 